\title{
Comparison of three different techniques of low-dose-rate seed implantation for prostate cancer
}

\author{
Hiromichi Ishiyama, MD!, Takefumi Satoh, MD², Akane Sekiguchi, MD!, Ken-ichi Tabata, MD², Shouko Komori, MD!, \\ Hideyasu Tsumura, MD², Shogo Kawakami, MD', Itaru Soda, MD', Kouji Takenaka, MD', Masatsugu Iwamura, MD², \\ Kazushige Hayakawa, MD' \\ 'Department of Radiology and Radiation Oncology, ${ }^{2}$ Department of Urology, Kitasato University School of Medicine, Kitasato, \\ Sagamihara, Japan
}

\begin{abstract}
Purpose: Three different techniques of low-dose-rate seed implantation for prostate cancer have been used since its use started in our hospital. The purpose of this study was to compare the results of the three different techniques.

Material and methods: The data of 305 prostate cancer patients who underwent low-dose-rate seed implantation were retrospectively analyzed. Pre-plan technique $(n=27)$, intraoperative pre-plan technique $(n=86)$, and interactive plan technique $(n=192)$ were tried in chronological order. The prescribed dose was set at $145 \mathrm{~Gy}$.

Results: Median follow-up was 66 months (range: 12-94 months). The 5-year biochemical control rate was 95.5\% (pre-plan group: 100\%, intraoperative pre-plan group: $90.7 \%$, interactive plan group: $97.0 \% ; p=0.08$ ). Dosimetric parameters were generally increased from the pre-plan group to the interactive group. The differences in some dosimetric parameters between the planning phase and the CT analysis were significantly reduced with the interactive plan compared to the other techniques. The interactive plan showed a significant reduction of the seed migration rate compared to the two other groups. Acute genitourinary toxicity, acute gastrointestinal toxicity, frequency, and urinary retention increased gradually from the pre-plan period to the interactive plan period.

Conclusions: There was no significant difference in biochemical control among the three groups. Dose-volume parameters were increased from the pre-plan technique to the interactive plan technique. However, this may not necessarily be due to technical improvements, since dose escalation was started during the same period. Lower seed migration rates and the smaller differences between the planning phase and CT analysis with the interactive plan technique suggest the superiority of this technique to the two other techniques.
\end{abstract}

Key words: interactive plan, intraoperative pre-plan, pre-plan, low-dose-rate, prostate cancer, seed implantation.

\section{Purpose}

Seed implantation for localized prostate cancer is one of the most important techniques that has been imported from foreign countries into Japan in this decade. This technique has spread rapidly throughout Japan and is now one of the standard treatments for localized prostate cancer. When this technique began to be used in Japan in 2004, there were several techniques already established in the United States and European countries [1,2], and it was necessary to identify the best technique for Japanese patients and physicians.

To date, we have tried three different techniques including the "pre-plan technique", the "intraoperative pre-plan technique", and the "interactive plan technique" in our hospital. Of these three techniques, we selected the interactive plan technique as the best for our patients, and over 1,000 patients have been treated with this technique. The purpose of this study was to analyze the results of the three different techniques, and to find which technique could lead to higher $\mathrm{D}_{90}$ or $\mathrm{V}_{100}$, or less toxicity, or lower seed migration rates than others.

\section{Material and methods}

\section{Patients}

Data of 305 consecutive Japanese prostate cancer patients who underwent low-dose-rate seed implantation between May 2004 and August 2007 were analyzed ret- 
Table 1. Patients' characteristics

\begin{tabular}{|c|c|c|c|c|}
\hline Factors & Pre-plan group & Intraoperative pre-plan group & Interactive plan group & $p$ \\
\hline$n$ & 27 & 86 & 192 & \\
\hline Age (years) & $69(5.5)$ & $68(6.3)$ & $68(6.0)$ & ns \\
\hline \multicolumn{5}{|l|}{ T stage } \\
\hline $1 \mathrm{c}$ & $17(63.0 \%)$ & $78(90.7 \%)$ & $156(81.3 \%)$ & \multirow[t]{4}{*}{$<0.001$} \\
\hline $2 a$ & $4(14.8 \%)$ & $8(9.3 \%)$ & $35(18.2 \%)$ & \\
\hline $2 b$ & $6(22.2 \%)$ & $0(0.0 \%)$ & $1(0.5 \%)$ & \\
\hline $2 c$ & $0(0.0 \%)$ & $0(0.0 \%)$ & $0(0.0 \%)$ & \\
\hline \multicolumn{5}{|l|}{ Gleason score } \\
\hline$\leq 3+3$ & $17(63.0 \%)$ & $63(73.3 \%)$ & $97(50.5 \%)$ & \multirow[t]{3}{*}{$<0.01$} \\
\hline $3+4$ & $5(18.5 \%)$ & $15(17.4 \%)$ & $70(36.5 \%)$ & \\
\hline $4+3$ & $5(18.5 \%)$ & $8(9.3 \%)$ & $25(13.0 \%)$ & \\
\hline Initial prostate-specific antigen $(\mathrm{ng} / \mathrm{ml})$ & $8.03(4.48)$ & $6.49(2.24)$ & $6.45(2.16)$ & ns \\
\hline Hormonal therapy & $9(33.3 \%)$ & $28(32.6 \%)$ & $56(29.2 \%)$ & ns \\
\hline
\end{tabular}

rospectively. The patients' characteristics are shown in Table 1. Although there was no clear reason, the pre-plan group included a slightly higher number of intermediate-risk patients with $\mathrm{T} 2 \mathrm{~b}$ or a Gleason score of $4+3$ $(p<0.01)$. Patients with clinical stage T1c or T2a who also had a prostate-specific antigen (PSA) level $\leq 10 \mathrm{ng} / \mathrm{ml}$ and a biopsy Gleason score (GS) of 2-6, have been defined as a low-risk group. Conversely, patients with clinical stage T2c or a PSA level $>20 \mathrm{ng} / \mathrm{ml}$ or a biopsy GS of $\geq 8$ have been defined as a high-risk group. The remaining patients have been defined as an intermediate-risk group. All patients in this study were low-risk or intermediate-risk patients.

Clinical staging was decided based on the results of digital rectal examinations and bone scintigraphy. Computed tomography (CT) and/or magnetic resonance imaging (MRI) of pelvis were also used to determine the T stage. Basically, hormonal manipulation was performed for three months in patients with a large prostate gland $\left(\geq 40 \mathrm{~cm}^{3}\right)$ before implantation. Gonadotropin-releasing hormone agonist with or without androgen blockade was used for hormonal manipulation. Some patients, however, received hormonal therapy before admission to our hospital.

\section{Pre-plan technique period}

In the first 27 patients (treated from May 2004 to October 2004), dosimetry was planned based on ultrasound (US) performed 4 weeks before implantation. Ultrasound images were acquired from transrectal ultrasonography in the extended lithotomy position. All dosimetry was planned using Interplant 3.2 software (CMS, St. Louis, MO, USA) with acquired US images as pre-planning. The prescribed dose to the prostate with a 3- to 5-mm margin was set at 145 Gy. Needle positions were symmetrical, and seeds were generally placed 5-mm apart from each other.

The procedure was performed in the extended lithotomy position. Seeds were placed one by one transperine- ally through needles attached to a Mick applicator (Mick Radio-Nuclear Instrument, Mount Vernon, NY, USA) under transrectal ultrasonography. Stranded seed was not used. All patients were hospitalized the day before seed implantation and discharged 2 days after implantation.

\section{Intraoperative pre-plan technique period}

In the next 86 patients (treated from October 2004 to October 2005), dosimetry was planned intraoperatively based on US performed just before implantation in the anesthetized patient. Using this technique, the problem of prostate volume change during the waiting time between pre-plan and operation could be resolved. Since dose escalation was started in this period, seeds were sequentially placed with no space if needed. The other procedures were the same as in the previous period.

\section{Interactive plan technique period}

In the remaining 192 patients (treated from October 2005 to August 2007), an interactive plan technique [3] was used. During this period, the prostate image was acquired after needle insertion. Therefore, prostate swelling and deformation were included in planning images. If needed, dosimetry was modified based on real-time dose calculation during the operation. The other procedures were the same as in the previous period.

\section{Follow-up}

Serum PSA levels were monitored every 3 months for the first year, and every 3-6 months thereafter. Biochemical failure was defined according to the Phoenix definition [4]. Urinary and rectal morbidities were assessed using the Radiation Therapy Oncology Group (RTOG) scale [5] and National Cancer Institute Common Toxicity Criteria (NCI-CTC) version 3. All patients received a-blockers just after seed implantation to relieve urinary symptoms. 


\section{Dosimetric analysis}

Post-implantation dosimetric analysis was performed using CT scans performed 30 days after implantation according to the recommendations of the American Brachytherapy Society [6]. Dose-volume histograms (DVHs) were calculated for each patient. Instead of actual urethral contouring, the surrogate urethra assumed to be at the geometric center of the prostate was contoured on the same slices as the prostate. The rectal wall was contoured, including the sphincter muscle, on the same slices as the prostate contouring.

\section{Statistics}

One-way analysis of variance or the Kruskal-Wallis test was used to compare the parameters of the three groups. The log-rank test was used to compare survival among the three groups. Stat Mate version 4.01 statistical software (ATMS, Tokyo, Japan) was used for data analysis. Differences were regarded as significant at the $p<0.05$ level.

\section{Results}

\section{Biochemical control}

Median follow-up was 66 months (range: 12-94 months). The 5-year biochemical control rate was $95.5 \%$ (pre-plan group: $100 \%$, intraoperative pre-plan group: $90.7 \%$, interactive plan group: $97.0 \% ; p=0.08$; Fig. 1). The 5-year clinical non-evidence of disease rate was $98.9 \%$ (pre-plan group: $100 \%$, intraoperative pre-plan group: $97.7 \%$, interactive plan group: $99.4 \%$ ). Two patients died of lung cancer, and the 5-year overall survival rate was $99.6 \%$. There were no significant differences among the three groups.

\section{Planning phase}

Table 2 shows comparisons of the three techniques in the planning phase. Interestingly, the numbers of seeds, needles, and total activity were increased when the technique was switched from pre-plan to intraoperative preplan $(p<0.01)$, and decreased when switched from intraoperative pre-plan to interactive plan $(p<0.01)$. Prostate volume was not different among the three groups. Dosimetric parameters, such as dose to $90 \%$ of prostate volume $\left(D_{90}\right)$, were generally increased from the pre-plan group to the interactive group. However, rectal volume receiving at least $150 \%$ of prescription dose $\left(\mathrm{RV}_{150}\right)$ was significantly reduced in the interactive plan group compared to the other two groups. The dose non-homogeneity ratio (DNR), which is the volume of $150 \%$ dose divided by the volume of the $100 \%$ dose, increased from pre-plan to interactive plan $(p<0.001)$.

\section{Computed tomography analysis}

Table 3 shows comparisons of the three techniques on the 1-month CT analysis. Dosimetric parameters such as $D_{90}$ (except $R V_{150}$ ) increased from the pre-plan group to the interactive group. Dose non-homogeneity ratio also increased from the pre-plan group to the interactive group. Seed migration rates were not different

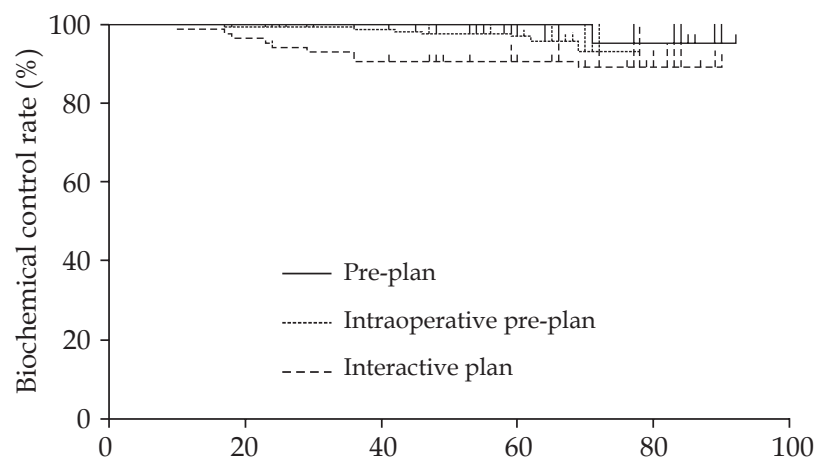

Fig. 1. Biochemical control rates of the pre-plan, intraoperative pre-plan, and interactive plan groups

between the pre-plan group and the intraoperative preplan group. However, the interactive plan group showed a significantly decreased seed migration rate compared to the other two groups $(p<0.001)$.

\section{Differences between the planning phase and computed tomography analysis}

Table 4 shows the differences in the DVH parameters between the planning phase and the 1-month CT analysis. When each corresponding parameter was compared, $\mathrm{D}_{90}$, prostate volume receiving at least $100 \%$ of the prescription dose $\left(\mathrm{V}_{100}\right)$, prostate volume receiving at least $150 \%$ of the prescription dose $\left(\mathrm{V}_{150}\right)$, and dose to $90 \%$ of urethral volume $\left(\mathrm{UD}_{90}\right)$ tended to decrease from the planning phase to the 1-month CT analysis. Meanwhile, rectal volume receiving at least $100 \%$ of the prescription dose $\left(\mathrm{RV}_{100}\right)$ and $\mathrm{RV}_{150}$ tended to be stable. Dose to $30 \%$ of urethral volume $\left(\mathrm{UD}_{30}\right)$ and dose to $10 \%$ of urethral volume $\left(\mathrm{UD}_{10}\right)$ tended to increase from the planning phase to the 1-month CT analysis.

The amount of the decrease for $V_{100}$ was significantly reduced from pre-plan to intraoperative pre-plan $(p<0.05)$, and it tended to be reduced from intraoperative pre-plan to interactive plan, but the difference was not significant. The amounts of the increases for $R V_{100}$ and $R V_{150}$ did not show significant differences between pre-plan and intraoperative pre-plan, but they showed a significant reduction from intraoperative pre-plan to interactive plan. These results suggest that the interactive plan technique helps achieve ideal dosimetry with a small difference between the planning phase and CT analysis compared to the other techniques.

\section{Toxicity}

Table 5 shows comparisons of toxicities among the three techniques. Acute morbidity was defined as morbidity occurring $\leq 12$ months after implantation, while late morbidity was defined as morbidity occurring $>12$ months after implantation. Acute genitourinary toxicity, acute gastrointestinal toxicity, frequency, and urinary retention increased gradually from the pre-plan period to the interactive plan period. The other toxicities were not different among the three groups. 
Table 2. Planning phase

\begin{tabular}{|c|c|c|c|c|}
\hline Factors & Pre-plan group & $\begin{array}{l}\text { Intraoperative pre-plan } \\
\text { group } \\
\end{array}$ & Interactive plan group & $p$ \\
\hline$n$ & 27 & 86 & 192 & \\
\hline Number of seeds & $84.1(16.54)$ & $97.5(14.3)$ & $89.4(15.4)$ & $<0.01$ \\
\hline Number of needles & $21.4(4.14)$ & $30.6(4.63)$ & $21.6(3.2)$ & $<0.001$ \\
\hline Total activity (MBq) & $1057.7(208.09)$ & $1226.7(180.31)$ & $1146.3(194.0)$ & $<0.01$ \\
\hline \multicolumn{5}{|l|}{ Prostate volume $(\mathrm{ml})$} \\
\hline US & $29.66(11.5)$ & $32.4(8.9)$ & $32.9(9.3)$ & ns \\
\hline 1-month CT & $29.08(12.54)$ & $27.0(8.0)$ & $28.4(8.7)$ & ns \\
\hline \multicolumn{5}{|l|}{ US plan } \\
\hline $\mathrm{D}_{90}(\mathrm{~Gy})$ & $168.2(11.13)$ & $184.0(11.08)$ & $192.7(9.4)$ & $<0.01$ \\
\hline$V_{100}(\%)$ & $97.4(1.87)$ & $99.6(0.49)$ & $99.5(0.6)$ & $<0.001$ \\
\hline $\mathrm{V}_{150}(\%)$ & $54.2(12.40)$ & $62.5(13.40)$ & $71.8(9.4)$ & $<0.001$ \\
\hline $\mathrm{RV}_{100}(\mathrm{ml})$ & $0.47(0.45)$ & $0.53(0.37)$ & $0.7(0.4)$ & $<0.01$ \\
\hline $\mathrm{RV}_{150}(\mathrm{ml})$ & $0.04(0.08)$ & $0.02(0.03)$ & $0.0(0.1)$ & $<0.05$ \\
\hline $\mathrm{UD}_{90}(\mathrm{~Gy})$ & $149.6(17.9)$ & $168.4(12.2)$ & $171.8(20.4)$ & $<0.001$ \\
\hline $\mathrm{UD}_{30}(\mathrm{~Gy})$ & $183.5(15.7)$ & $188.5(10.8)$ & $200.0(9.5)$ & $<0.001$ \\
\hline $\mathrm{UD}_{10}(\mathrm{~Gy})$ & $189.7(14.90)$ & $194.4(10.26)$ & $208.3(11.0)$ & $<0.001$ \\
\hline DNR & $0.56(0.13)$ & $0.63(0.13)$ & $0.72(0.09)$ & $<0.001$ \\
\hline
\end{tabular}

Values are given as means (standard deviation) or numbers.

$D_{90}$ - dose to $90 \%$ of prostate volume; $V_{100}$ - prostate volume receiving at least $100 \%$ of prescription dose; $V_{150}$ - prostate volume receiving at least $150 \%$ of prescription dose; $R V_{100}$ - rectal volume receiving at least $100 \%$ of prescription dose; $R V_{150}$ - rectal volume receiving at least $150 \%$ of prescription dose; $U D_{90}$ - dose to $90 \%$ of urethral volume; $U D_{30}$ - dose to $30 \%$ of urethral volume; $U D_{10}$-dose to $10 \%$ of urethral volume; US - ultrasound; DNR-dose non-homogeneity ratio

\section{Discussion}

The present results showed a favorable biochemical control rate for Japanese prostate cancer patients, although more biochemical failures will occur when the follow-up data are updated. The detailed analysis of biochemical control and its predictive factors were reported in our previous paper [7]. This study focused on the differences among the three techniques.

Regarding biochemical control, Shah et al. reported the superiority of the intraoperative pre-plan technique to the pre-plan technique [8]. Their 4-year biochemical control rates were $96 \%$ and $82 \%$ for the intraoperative preplan and pre-plan groups, respectively. Meanwhile, the present results showed a lower biochemical control rate for the intraoperative pre-plan group compared to the other groups, although the difference was not significant $(p=0.08)$. Additional multivariate analysis including initial PSA, Gleason score (these parameters were identified as significant variables in our previous paper), and the type of planning techniques was also performed. It also did not show that type of planning technique was significant (data not shown). Therefore, it seems that some confounding factors caused relatively low biochemical control of the intraoperative pre-plan group in this study. In addition, our follow-up time was too short to draw a conclusion regarding biochemical control. Because preplan group had lower $\mathrm{D}_{90}$ value compared to the other groups (Table 3), longer follow-up might reveal its lower biochemical control compared to the other groups.
Regarding DVH parameters, the dose of the planning phase was directly correlated to that of the 1-month CT analysis in the present study. The increased DVH parameters from pre-plan to interactive plan represented our dose-escalation policy that started in the intraoperative pre-plan period. The landmark paper of Stock et al. that reported dose dependency of biochemical failure [9] strongly encouraged our dose-escalation policy. As shown in Table 2, $\mathrm{D}_{90}$ increased gradually from the preplan group to the interactive plan group, because it has been suggested that a higher dose leads to better biochemical control. The upper limit was set at about $200 \mathrm{~Gy}$. Therefore, the improvement of DVH parameters may not necessarily be due to technical improvements, although our technical improvements must have had some effects. Several studies reported the superiority of intraoperative pre-plan to pre-plan $[10,11]$, and the superiority of interactive plan to pre-plan $[12,13]$. However, these studies were retrospective analyses and may have had some biases, such as dose-escalation policy, as in the present study.

Meanwhile, as the present study demonstrated, toxicity was increased with dose escalation, especially urinary frequency and urinary retention. However, only acute toxicity showed a significant difference but late toxicity and grade 3 toxicities were at acceptable levels. Matzkin et al. reported increased urinary toxicity from pre-plan to intraoperative pre-plan [14]. They reported a higher International Prostate Symptom Score (IPSS) in the intraoperative pre-plan group than in the pre-plan group. 
Table 3. Computed tomography at 1 month

\begin{tabular}{lcccc} 
Factors & Pre-plan group & $\begin{array}{c}\text { Intraoperative pre-plan } \\
\text { group }\end{array}$ & Interactive plan group & $p$ \\
\hline$n$ & 27 & 86 & 192 & $<0.001$ \\
\hline $\mathrm{D}_{90}(\mathrm{~Gy})$ & $145.0(33.9)$ & $169.4(23.2)$ & $179.1(21.8)$ & $<0.001$ \\
\hline $\mathrm{V}_{100}(\%)$ & $87.9(11.9)$ & $95.7(5.7)$ & $97.0(3.3)$ & $<0.01$ \\
\hline $\mathrm{V}_{150}(\%)$ & $51.7(16.0)$ & $60.1(15.4)$ & $68.5(13.9)$ & $<0.05$ \\
\hline $\mathrm{RV}_{100}(\mathrm{ml})$ & $0.8(0.7)$ & $1.16(1.0)$ & $0.1(0.2)$ & $<5$ \\
\hline $\mathrm{RV}_{150}(\mathrm{ml})$ & $0.1(0.2)$ & $0.15(0.2)$ & $161.5(28.7)$ & $<0.01$ \\
\hline $\mathrm{UD}_{90}(\mathrm{~Gy})$ & $133.3(42.1)$ & $155.1(25.4)$ & $218.5(26.1)$ & $<0.001$ \\
\hline $\mathrm{UD}_{30}(\mathrm{~Gy})$ & $194.0(29.5)$ & $192.6(20.7)$ & $234.6(31.9)$ & $<0.001$ \\
\hline $\mathrm{UD}_{10}(\mathrm{~Gy})$ & $209.7(36.4)$ & $218.3(31.5)$ & $0.70(0.13)$ & $<0.01$ \\
\hline DNR & $0.58(0.13)$ & $0.62(0.14)$ & $77(40.1 \%)$ & $<0.001$ \\
\hline Patients with migration $(1 \mathrm{mon})$ & $19(70.4 \%)$ & $60(69.8 \%)$ & $42(21.9 \%)$ & $<0.001$ \\
\hline Total & $14(51.9 \%)$ & $47(54.7 \%)$ & $41(21.4 \%)$ & $<0.001$
\end{tabular}

Values are given as means (standard deviation or percentage) or numbers.

Abbreviations are the same as in Table 2.

Table 4. Differences between the planning phase and the 1-month computed tomography analysis

\begin{tabular}{|c|c|c|c|c|}
\hline Factors & Pre-plan group & $\begin{array}{c}\text { Intraoperative pre-plan } \\
\text { group }\end{array}$ & Interactive plan group & $p$ \\
\hline$n$ & 27 & 86 & 192 & \\
\hline$D_{90}(G y)$ & $-23.2(32.0)$ & $-14.8(24.2)$ & $-12.6(20.4)$ & ns \\
\hline$V_{100}(\%)$ & $-9.5(11.4)$ & $-4.0(5.7)$ & $-2.4(3.3)$ & $<0.001$ \\
\hline$\bigvee_{150}(\%)$ & $-2.5(17.0)$ & $-0.2(21.8)$ & $-2.0(14.7)$ & ns \\
\hline $\mathrm{RV}_{100}(\mathrm{ml})$ & $0.3(0.7)$ & $0.60(0.9)$ & $-0.4(1.1)$ & $<0.01$ \\
\hline $\mathrm{RV}_{150}(\mathrm{ml})$ & $0.1(0.2)$ & $0.11(0.2)$ & $0.0(0.2)$ & $<0.05$ \\
\hline $\mathrm{UD}_{90}(\mathrm{~Gy})$ & $-16.3(40.1)$ & $-13.7(25.6)$ & $-8.7(31.6)$ & ns \\
\hline $\mathrm{UD}_{30}(\mathrm{~Gy})$ & $10.5(26.6)$ & $14.2(27.5)$ & $19.9(26.1)$ & ns \\
\hline $\mathrm{UD}_{10}(\mathrm{~Gy})$ & $20.0(31.6)$ & $20.5(39.4)$ & $29.6(32.5)$ & ns \\
\hline
\end{tabular}

Values are given as means (standard deviation) or numbers.

Abbreviations are the same as in Table 2.

However, they also reported increased DVH parameters such as $\mathrm{D}_{90}$ and urethral $\mathrm{V}_{150}$, as in the present study, and their increased toxicity may not have come from technical changes but increased prostate or urethral doses. Keyes et al. reported number of needles as one of predictive factors of acute urinary toxicity [15]. Although our number of needles was temporarily increased during intraoperative pre-plan, it decreased during interactive plan technique (Table 2). In the same period, acute urinary toxicity was steadily increased (Table 5). Therefore, the number of needles did not seem to have a significant effect on urinary toxicity at least in our study population.

Regarding seed migration, the present study results suggest that the interactive plan technique can reduce the seed migration rate. Stone et al. already reported that the interactive technique can reduce the seed migration rate [16], and the present study confirmed their report. Meanwhile, stranded-seed or linked-seed were suggested to have a strong impact on seed migration rate. Our recent publication reveals that stranded-seed or linked-seed can reduce seed migration rate significantly without compromising dosimetric parameters [17].

The learning curve of our brachytherapy team was one of the important factors related to our dosimetric results. Lower seed migration rates and the smaller DVH differences between the planning phase and CT analysis may suggest some technical improvements of our brachytherapy team. The personal learning curve of each team member was another important factor. Our brachytherapy team, however, consisted of at least 
Table 5. Crude toxicity rates

\begin{tabular}{|c|c|c|c|c|}
\hline Factors & Pre-plan group & Intraoperative pre-plan group & Interactive plan group & $p$ \\
\hline$n$ & 27 & 86 & 192 & \\
\hline \multicolumn{5}{|l|}{ RTOG GU acute } \\
\hline Grade 1 & $19(70 \%)$ & $56(65 \%)$ & 119 (62\%) & \multirow[t]{3}{*}{$<0.01$} \\
\hline Grade 2 & $1(4 \%)$ & $12(14 \%)$ & $50(26 \%)$ & \\
\hline Grade 3 & $0(0 \%)$ & $4(5 \%)$ & $10(5 \%)$ & \\
\hline \multicolumn{5}{|l|}{ RTOG GU late } \\
\hline Grade 1 & $13(48 \%)$ & $53(62 \%)$ & $108(56 \%)$ & \multirow[t]{3}{*}{ ns } \\
\hline Grade 2 & $1(4 \%)$ & $2(2 \%)$ & $18(9 \%)$ & \\
\hline Grade 3 & $0(0 \%)$ & $3(3 \%)$ & $3(2 \%)$ & \\
\hline \multicolumn{5}{|l|}{ RTOG GI acute } \\
\hline Grade 1 & $2(7 \%)$ & $8(9 \%)$ & $44(23 \%)$ & \multirow[t]{2}{*}{$<0.05$} \\
\hline Grade 2 & $1(4 \%)$ & $1(1 \%)$ & $2(1 \%)$ & \\
\hline \multicolumn{5}{|l|}{ RTOG GI late } \\
\hline Grade 1 & $6(22 \%)$ & $18(21 \%)$ & $46(24 \%)$ & \multirow[t]{3}{*}{ ns } \\
\hline Grade 2 & $0(0 \%)$ & $1(1 \%)$ & $2(1 \%)$ & \\
\hline Grade 3 & $0(0 \%)$ & $0(0 \%)$ & $1(1 \%)$ & \\
\hline \multicolumn{5}{|l|}{ Micturition pain } \\
\hline Grade 1 & $10(37 \%)$ & $37(43 \%)$ & $83(43 \%)$ & \multirow[t]{2}{*}{ ns } \\
\hline Grade 2 & $0(0 \%)$ & $3(3 \%)$ & $8(4 \%)$ & \\
\hline \multicolumn{5}{|l|}{ Proctitis } \\
\hline Grade 1 & $6(22 \%)$ & $8(9 \%)$ & $34(18 \%)$ & \multirow[t]{2}{*}{ ns } \\
\hline Grade 2 & $1(4 \%)$ & $0(0 \%)$ & $1(1 \%)$ & \\
\hline \multicolumn{5}{|c|}{ Incontinence (stool) } \\
\hline Grade 1 & $1(4 \%)$ & $0(0 \%)$ & $1(1 \%)$ & \multirow[t]{2}{*}{ ns } \\
\hline Grade 2 & $0(0 \%)$ & $0(0 \%)$ & $0(0 \%)$ & \\
\hline \multicolumn{5}{|l|}{ Diarrhea } \\
\hline Grade 1 & $1(4 \%)$ & $6(7 \%)$ & $5(3 \%)$ & \multirow[t]{2}{*}{ ns } \\
\hline Grade 2 & $0(0 \%)$ & $1(1 \%)$ & $0(0 \%)$ & \\
\hline \multicolumn{5}{|l|}{ Rectal bleeding } \\
\hline Grade 1 & $3(11 \%)$ & $19(22 \%)$ & 47 (24\%) & \multirow[t]{2}{*}{ ns } \\
\hline Grade 2 & $0(0 \%)$ & $1(1 \%)$ & $3(2 \%)$ & \\
\hline \multicolumn{5}{|l|}{ Frequency } \\
\hline Grade 1 & $19(70 \%)$ & $55(64 \%)$ & $99(52 \%)$ & \multirow[t]{3}{*}{$<0.01$} \\
\hline Grade 2 & $1(4 \%)$ & $15(17 \%)$ & $58(30 \%)$ & \\
\hline Grade 3 & $0(0 \%)$ & $0(0 \%)$ & $6(3 \%)$ & \\
\hline \multicolumn{5}{|c|}{ Incontinence (urine) } \\
\hline Grade 1 & $0(0 \%)$ & $5(6 \%)$ & $3(2 \%)$ & \multirow[t]{2}{*}{ ns } \\
\hline Grade 2 & $1(4 \%)$ & $1(1 \%)$ & $4(2 \%)$ & \\
\hline Urinary retentior & & & & \\
\hline Grade 1 & $10(37 \%)$ & $50(58 \%)$ & 137 (71\%) & $<0.01$ \\
\hline Grade 2 & $1(4 \%)$ & $2(2 \%)$ & $10(5 \%)$ & \\
\hline Grade 3 & $0(0 \%)$ & $4(5 \%)$ & $7(4 \%)$ & \\
\hline Hematuria & & & & \\
\hline Grade 1 & $4(15 \%)$ & $11(13 \%)$ & $29(15 \%)$ & ns \\
\hline Grade 2 & $1(4 \%)$ & $0(0 \%)$ & $5(3 \%)$ & \\
\hline Stricture & & & & \\
\hline Grade 1 & $0(0 \%)$ & $1(1 \%)$ & $1(1 \%)$ & ns \\
\hline Grade 2 & $0(0 \%)$ & $0(0 \%)$ & $0(0 \%)$ & \\
\hline Grade 3 & $0(0 \%)$ & $1(1 \%)$ & $0(0 \%)$ & \\
\hline
\end{tabular}

Values are given as means (standard deviation) or numbers.

RTOG - Radiation Therapy Oncology Group; GU - genitourinary; GI - gastrointestinal 
4 urologists and 3 radiation oncologists during the study period, and it was difficult to conduct separate analyses in our database.

In our planning phase, the numbers of seeds and needles and total activity were increased when the technique was switched from pre-plan to intraoperative pre-plan, and decreased when switched from intraoperative preplan to interactive plan (Table 2). Our dose escalation policy might have increased these numbers, and then our technical improvements, including efficient seed positioning, might have decreased these numbers.

\section{Conclusions}

In conclusion, the present study showed that DVH parameters improved from the pre-plan technique to the interactive plan technique. However, this improvement may not necessarily be due to technical improvements, because dose escalation was started during the same period. Lower seed migration rates and the smaller DVH differences between the planning phase and the CT analysis in the interactive plan period suggest the superiority of the interactive plan to the two other techniques.

\section{Disclosure}

This work was supported by JSPS KAKENHI Grant Number 24791334.

H. Ishiyama, T. Satoh and K. Hayakawa received honoraria for lecture fees from Medicon Co., Ltd. and Nihon Medi-Physics Co., Ltd.

\section{References}

1. Aronowitz JN, Rivard MJ. The phylogeny of permanent prostate brachytherapy. J Contemp Brachytherapy 2013; 5: 89-92.

2. Skowronek J. Low-dose-rate or high-dose-rate brachytherapy in treatment of prostate cancer - between options. J Contemp Brachytherapy 2013; 5: 33-41.

3. Stock RG, Stone NN, Wesson MF et al. A modified technique allowing interactive ultrasound-guided three-dimensional transperineal prostate implantation. Int J Radiat Oncol Biol Phys 1995; 32: 219-225.

4. Roach M, 3rd, Hanks G, Thames H Jr. et al. Defining biochemical failure following radiotherapy with or without hormonal therapy in men with clinically localized prostate cancer: Recommendations of the rtog-astro phoenix consensus conference. Int J Radiat Oncol Biol Phys 2006; 65: 965-974.

5. Cox JD, Stetz J, Pajak TF. Toxicity criteria of the Radiation Therapy Oncology Group (RTOG) and the European Organization for Research and Treatment of Cancer (EORTC). Int J Radiat Oncol Biol Phys 1995; 31: 1341-1346.

6. Nag S, Beyer D, Friedland J et al. American Brachytherapy Society (ABS) recommendations for transperineal permanent brachytherapy of prostate cancer. Int J Radiat Oncol Biol Phys 1999; 44: 789-799.

7. Sekiguchi A, Ishiyama H, Satoh $\mathrm{T}$ et al. 125iodine monotherapy for Japanese men with low- and intermediate-risk prostate cancer: Outcomes after 5 years of follow-up. J Rad Res 2014; 55: 328-333.

8. Shah JN, Wuu CS, Katz AE et al. Improved biochemical control and clinical disease-free survival with intraoperative versus preoperative preplanning for transperineal interstitial permanent prostate brachytherapy. Cancer J 2006; 12: 289-297.
9. Stock RG, Stone NN, Cesaretti JA et al. Biologically effective dose values for prostate brachytherapy: Effects on PSA failure and posttreatment biopsy results. Int J Radiat Oncol Biol Phys 2006; 64: 527-533.

10. Wilkinson DA, Lee EJ, Ciezki JP et al. Dosimetric comparison of pre-planned and or-planned prostate seed brachytherapy. Int J Radiat Oncol Biol Phys 2000; 48: 1241-1244.

11. Matzkin H, Kaver I, Bramante-Schreiber L et al. Comparison between two iodine-125 brachytherapy implant techniques: Pre-planning and intra-operative by various dosimetry quality indicators. Radiother Oncol 2003; 68: 289-294.

12. Shanahan TG, Nanavati PJ, Mueller PW et al. A comparison of permanent prostate brachytherapy techniques: preplan vs. hybrid interactive planning with postimplant analysis. Int J Radiat Oncol Biol Phys 2002; 53: 490-496.

13. Zelefsky MJ, Yamada Y, Cohen G et al. Postimplantation dosimetric analysis of permanent transperineal prostate implantation: improved dose distributions with an intraoperative computer-optimized conformal planning technique. Int J Radiat Oncol Biol Phys 2000; 48: 601-608.

14. Matzkin H, Kaver I, Stenger A et al. Iodine-125 brachytherapy for localized prostate cancer and urinary morbidity: a prospective comparison of two seed implant methods-preplanning and intraoperative planning. Urology 2003; 62: 497-502.

15. Keyes M, Miller S, Moravan V et al. Predictive factors for acute and late urinary toxicity after permanent prostate brachytherapy: long-term outcome in 712 consecutive patients. Int J Radiat Oncol Biol Phys 2009; 73: 1023-1032.

16. Stone NN, Stock RG. Reduction of pulmonary migration of permanent interstitial sources in patients undergoing prostate brachytherapy. Urology 2005; 66: 119-123.

17. Ishiyama $H$, Satoh $T$, Kawakami $S$ et al. A prospective quasi-randomized comparison of intraoperatively built custom-linked seeds versus loose seeds for prostate brachytherapy. Int J Radiat Oncol Biol Phys 2014; 90: 134-139. 